\title{
What can we learn from minority-serving institutions?
}

\author{
Apriel K. Hodari \\ Resource Analysis Division, The CNA Corporation, Alexandria, VA 22311 \\ Jeffery M. Saul \\ Department of Physics, University of Central Florida, Orlando, FL 32816 \\ Beth Hufnagel \\ Department of Astronomy/Chemistry/Physical Science/Physics \\ Anne Arundel Community College, Arnold, MD 21012
}

\begin{abstract}
Despite the decades since the Civil Rights movement, women and minorities are still greatly under-represented in mainstream science education and practice, with physics being one of the fields of lowest participation. In the face of this, 84 schools do successfully serve under-represented minorities and women. These 84 schools include 34 Historically Black Colleges and Universities (HBCUs) that grant $60 \%$ of all physics bachelors earned by African-Americans. ${ }^{1}$ Similarly, 16 Women's Colleges and Universities (WCUs) grant $5 \%$ of such degrees earned by women. ${ }^{2}$ It is our thesis that the 84 institutions we are targeting have something to teach the rest of us about promoting the success of under-represented minorities and women. ${ }^{3}$ Based on our previous research ${ }^{4}$ of introductory physics courses at HBCUs and WCUs, we propose two separate projects to carefully examine these institutions for admissions, curricular, and environmental practices that could improve majority institutions' ability to promote the success of women and minorities in physics. This paper discusses the background to our proposals, and lists key features of the proposed research. We seek input regarding our focus, experimental design, and research methodologies.
\end{abstract}

\section{INTRODUCTION}

Scientists operate within a tradition of discovering, constructing and applying understandings of the physical world by engaging in scholarly debate, working rigorously to refine ideas, and pushing forward the boundaries of knowledge. Within this framework, academic scientists have constructed methods for educating students, with the belief that the scientific approach is applicable to many human pursuits, and the hope that some students will rise to the challenges of the scientific disciplines, and become scientists themselves. As teachers, many scientists believe that these education and training processes are wisely conceived, justly applied and produce the correct results.

Despite this proud tradition, the academy is now perceived as not having produced a just result for most of the students in science classrooms, particularly since few of them will pursue traditional research careers. In response to this perception, some scientists now focus on improving the learning by the majority of their students, rather than the few who learn like them.
Notwithstanding this progress, the lagging success of under-represented minorities and women in science classrooms has not been adequately addressed. In the research described below, we focus on the factors that disproportionately impact under-represented minorities and women, and specifically investigate the success of minority-serving institutions with these populations. We propose to study these institutions for ways majority schools can promote the success of the women and minorities in their classrooms.

We focus on the introductory physics course, since it is both the gateway to further physics study and an important foundation course for students intending to study other sciences, engineering, and medicine.

\section{BACKGROUND}

The research of Perry ${ }^{5}$ and Belenky et $a l^{6}$ suggests that it is crucial for educators to understand and allow for differing stages of intellectual development and academic maturity in the students they teach. By applying these ideas to introductory physics learning, Hammer, ${ }^{7}$ Redish and Steinberg, ${ }^{8}$ Saul, ${ }^{9}$ and 
Elby $^{10}$ have found that what students believe about the nature of scientific knowledge and how they acquire that knowledge are as important as the content they are learning. Further, researchers have shown that student epistemologies, and the conflicts between them and what students perceive of instructor epistemologies, can profoundly influence the amount and quality of learning that takes place in the classroom. ${ }^{11}$ In practical terms, this means that students often use different strategies to learn science than do expert scientists. ${ }^{12}$

Physics education researchers sometimes refer to the collection of epistemic beliefs that students have about learning as expectations. Based on the work of Perry, Belenky, and Hammer $^{13}$, the Physics Education Research Group at the University of Maryland developed the Maryland Physics Expectations (MPEX) Survey. The MPEX Survey contains 34 statements, to which respondents are asked to agree or disagree on a five-point Likert scale. This format allows researchers and instructors to probe the expectations of large numbers of students, in order to gain insight into the effect of different kinds of physics instruction on the students' epistemic beliefs.

The Maryland researchers collected and analyzed a databank of 4000 matched sets of MPEX data from students at 17 different institutions around the country. Their results showed a deterioration of student expectations for all classes in which traditional lecture-labrecitation instruction was used, regardless of institution size or type. ${ }^{14}$ In very few cases did student expectations hold steady or improve, and these few cases involved intensive, research-based, inquiry curricula that required substantive changes in the course structure and instructional pedagogy. ${ }^{15}$

In the same study, data on student conceptual understanding of Newtonian Mechanics was collected. Both the Force Concept Inventory (FCI) ${ }^{16}$ and the Force and Motion Conceptual Evaluation (FMCE) ${ }^{17}$ were used. FCI/FMCE data from over 9000 student respondents were collected in two different studies, and consistently showed better results due to interactive engagement instruction as compared to traditional instruction.

Despite the collection of these large data sets, no students at HBCUs and few at WCUs were surveyed. These omissions inspired the formation of the Hodari-Hufnagel-Saul collaboration to investigate HBCUs and WCUs. The overall results of the HBCU/WCU project are consistent with those found by earlier researchers, with a few interesting exceptions. (See Figure 1. HBCU/WCU data shown in solid colors.) Like the schools in the previous study, the schools in the HBCU/WCU study using traditional (TRD) instruction produced MPEX scores which deteriorated after instruction. These traditionally-taught courses also produced FMCE gains comparable to traditionally-taught courses surveyed in the Maryland study.



Figure 1. FMCE gains, $h$, depend on type of instruction, not type of institution.

Our work has also been informed by the work of social psychologists. We have been particularly influenced by the groundbreaking work of Claude Steele and colleagues, who named a phenomenon they call stereotype threat. ${ }^{18}$ Steele defines stereotype threat as the psychological threat a subject feels when he/she believes that a stereotype may separate him/her from his/her chosen academic community. This threatened state causes the subject to underperform as compared to otherwise welldocumented capability. One key feature of stereotype threat is the subject's belief that she or he is a legitimate member of said community and that a particular compulsory activity may separate her or him from this community based on an existing social stereotype. ${ }^{19}$

Steele et al have tested this theory in several ways. In one of their first experiments, they selected two academically matched groups of Stanford University sophomore mathematics majors and placed them in groups containing 
both men and women. Next they administered a test comprised of the twenty most difficult items from the Mathematics Graduate Record Examination (GRE). The experimental group was told that the test tended to separate people by gender. The control group was told that the test was experimental, and that they should just try to do their best. This experiment operated on the stereotype that men do better in math than women. As is shown in Figure $2,{ }^{20}$ women in the threatened group vastly under-performed as compared to women in the unthreatened group. Conversely, men over-performed in the threatened group.

Steele's group recorded similar underperformance by African-American English majors by doing an equivalent experiment on groups of African-American and EuropeanAmerican students, using items from the Verbal GRE, based on the stereotype that AfricanAmericans have inferior verbal skills. They then probed the subtlety of the result by repeating the verbal experiment and simply asking the students to check off their race before beginning the test. The fact that the latter experiment produced the same results may explain the under-performance of women and minorities on standardized tests, particularly in light of the routine collection of demographic information just prior to test administration.

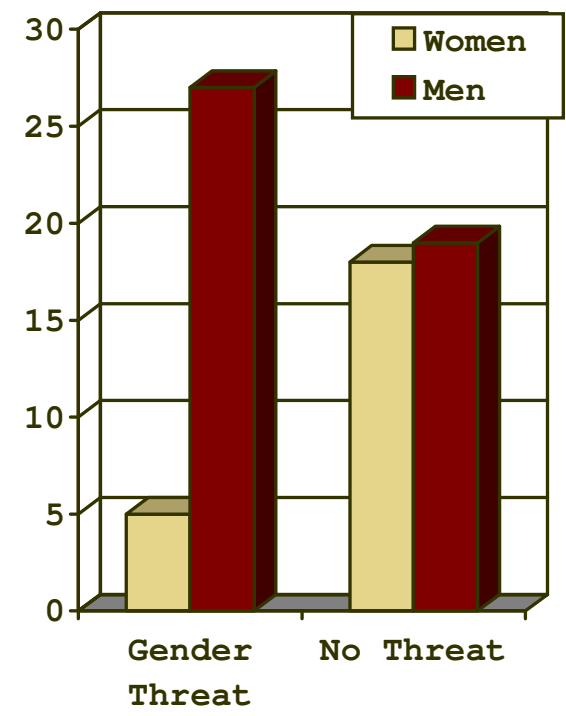

Figure 2. Steele data on gender threat.
In the HBCU/WCU project, Steele's results were applied to our methods of data collection. To avoid the potential of stereotype threat, we did not collect any demographic data on survey respondents until after both pre-course and postcourse data was collected. While the very presence of spaces for demographic data on standardized data sheets may also have an effect, we did not have the resources to employ efficient alternative means for data collection at that time.

Novel research on the connection between sports participation and physics understanding has also informed our work. Melissa Dancy compared sports participation and performance on a standardized conceptual physics test. ${ }^{21}$ She found a high correlation between certain kinds of sports participation and pre-course performance on the Force Concept Inventory (FCI) for women but no correlation at all for men. Further, Sandra Hanson found a similar correlation for European-American women and no correlation for African-American women. ${ }^{22}$

In sum, previous work has provided a number of hypotheses that can be tested.

- What students believe about the nature of scientific knowledge and how they acquire that knowledge is as important as the knowledge itself.

- The MPEX may point to key features of educational environments that promote the success of women and minorities in physics.

- The FMCE may provide evidence of successful curricular practices used in non-traditional settings.

- Stereotype threat may account for the differential performance between minorities and women in traditional and non-traditional settings. Eliminating stereotype threat in both institutional culture and test administration may be a key step toward accurately assessing the performance of women and minority physics students.

- There may be a connection between sports participation and understanding of conceptual physics for some women.

These hypotheses will guide the selection of research instruments used and methodologies. 


\section{STUDYING WHAT WORKS}

Overall, we approach this work from the perspective that the 48 institutions we are targeting are doing something right. In most cases, these schools operate with fewer resources and garner more modest reputations than do top-tier schools, yet they manage to far outpace more prominent institutions in promoting the academic success of marginalized populations. Further, some evidence has been gathered which shows that graduates from these institutions attend graduate schools at impressive rates. ${ }^{23}$

We hypothesize that this success is not accidental, but that it results from pervasive institutional commitments to serving these populations well. We expect to find fundamental elements of this success throughout these organizations: in their admissions processes; in their orientation, placement and counseling activities; in their mechanisms for both academic and non-academic support; and in the interactions students have with faculty, staff and administrators.

While we intend to conduct two separate studies, one on gender equity and the other on representational equity, several activities will be common to both. In both studies we will investigate student learning using pre-post testing; classroom observations; and student, faculty and administrator interviews. We will test for the presence of stereotype threat in our survey instruments and work to eliminate it. We will develop and make use of stringent privacy protect mechanisms, to encourage full and candid responses, and to protect our participants from the exploitation many of them have already experienced. Lastly, our research team will include experts in social psychology, minority and female education, and innovative statistical analysis techniques.

\section{TOWARD GENDER EQUITY}

Research and anecdotal evidence has shown that women prefer collaborative atmospheres rather than competitive ones. ${ }^{24}$ Therefore, we may find that physics classrooms that are successful for women may encourage students to work collaboratively, and explicitly remove activities that foster a competition. Perhaps such environmental factors will eventually close the gap between the $50 \%$ female participation in high school physics courses and the less than
$20 \%$ graduation rates of women in physics and engineering. ${ }^{25}$

Conducting research on schools that successfully promote the success of women will also give us a chance to investigate the source of the expert-level MPEX results produced by students at one of the WCUs previously studied. With this study we will be able to discern whether the previous result was an isolated incident or whether mature expectations are systematically linked to instructional practice or institutional culture at certain schools.

\section{TOWARD REPRESENTATIONAL EQUITY}

Research has also shown that African-American students perform better when they have both academic and non-academic support. ${ }^{26}$ If creating a well-integrated living and learning environment is found to be key, then perhaps we will find that the schools that produce the majority of African-American bachelor physics degrees foster this environment successfully.

\section{NEXT STEPS}

While we are awaiting the results of our recently submitted funding proposals, we will begin recruiting participants and expanding our research team. We invite comments, questions and critiques as we develop our research plan.

\section{REFERENCES}

1. P. J. Mulvey, Private communication (May, 2001).

2. R. Ivie and K. Stowe, Women in Physics, 2000, A Report to the American Institute of Physics (2000).

3 . Included in the targeted schools are 20 coeducational schools that graduate bachelor physics cohorts containing $40 \%$ or more women, and 14 non-HBCUs that graduate at least $15 \%$ African-Americans.

4. A. K. Hodari, B. Hufnagel and J. Saul, "Student cognitive expectations in nontraditional settings," Announcer 29, 78 (Winter 1999); Partially supported by NSF Grants NGE 9714452 and DGE 9714489.

5. W. G. Perry, Jr., Forms of ethical and intellectual development in the college years: A scheme (originally published by Holt, Rinehart Winston, Inc., 1968).

6. M. F. Belenky, B. M. Clinchy, N. R. Goldberger and J. M. Tarule, Women's ways of knowing: The development of self, voice, and mind (Basic Books, 1986). 
7. D. Hammer, "Epistemological beliefs in introductory physics," Cognition and Instruction 12(2), 151-183 (1994).

8. E. F. Redish and R. N. Steinberg, "Teaching physics: Figuring out what works," Physics Today 52(1), 24-30 (1999).

9. J. M. Saul, Beyond problem solving: Evaluating introducing physics courses through the hidden curriculum, University of Maryland, College Park, Maryland, Ph. D. Dissertation (1998).

10. A. Elby, "Helping physics students learn about learning," Physics Education Research Supplement to the American Journal of Physics 69(7SUPP1), in press (2001).

11. D. Hammer, "Epistemological consideration in teaching introductory physics," Science Education 79(4), 393413 (1995).

12. D. Hammer, "Two approaches to learning physics," The Physics Teacher 27, 664-671 (1989); A. Elby, "Another reason that physics students learn by rote," Physics Education Research Supplement to the American Journal of Physics 67(7SUPP1), S52-S57 (1999).

13. D. M. Hammer, Defying common sense: Epistemological beliefs in an introductory physics course, University of California at Berkeley, Berkeley, California, Ph. D. Dissertation (1991).

14. Saul (1998).

15. Saul (1998).

16. D. Hestenes, M. Wells and G. Swackhammer, "Force Concept Inventory," The Physics Teacher 30, 141158 (1992).

17. R. K. Thornton and D. R. Sokoloff, "Assessing student learning of Newton's laws: The Force and Motion Conceptual Evaluation," American Journal of Physics 66(4), 338-351 (1998).
18. C. M. Steele, "A threat in the air," American Psychologist 52(6), 613-629 (1997).

19. C. Steele, "Thin ice: 'Stereotype threat' and Black college students," Atlantic Monthly (1999).

20. Steele (1997).

21. M. H. Dancy and R. J. Beichner, "The relationship between sports playing and performance on the Force Concept Inventory for men and women," Announcer 29(4), 77 (1999).

22. S. L. Hanson and E. P. Johnson, "African American women in science: Expecting the unexpected," Paper presented at The American Sociological Association Meeting, Toronto, Ontario (August 1997).

23. F. A. Hrabowski, Beating the odds: Raising academically successful African American males (Oxford University Press, 1998); F. A. Hrabowski, G. Greif, K. Maton and M. Greene Overcoming the odds: Raising academically successful African American young women In press (Oxford University Press, 2002).

24. S. Tobias, They're not dumb, they're different: Stalking the second tier (Research Corporation, 1990); E. Seymour and N. M. Hewitt, Talking about leaving: Why undergraduates leave the sciences (Westview Press, 1997); R. Lippmann, Private communication (1999).

25. Ivie and Stowe (2000).

26. P. U. Treisman, "Improving the performance of minority students in collegelevel mathematics," Innovative Abstracts V(17), 3-4 (1983); Hrabowski (1998); Hrabowski, Greif, Maton and Greene (2002). 